\title{
Designing of active plasma lens for focusing laser-plasma-accelerated pulsed proton beams
}

\author{
Tong Yang $\odot$, Hao Cheng, Yang Yan, Minjian Wu®, Dongyu Li, Yuze Li, and Yadong Xia \\ State Key Laboratory of Nuclear Physics and Technology, Peking University, Beijing, 100871, China \\ and Beijing Laser Acceleration Innovation Center, Huairou, Beijing,101400, China \\ Chen Lin ${ }^{*}$ and Xueqing Yan \\ State Key Laboratory of Nuclear Physics and Technology, Peking University, Beijing, 100871, China; \\ Beijing Laser Acceleration Innovation Center, Huairou, Beijing, 101400, China; \\ and Institute of Guangdong Laser Plasma Techology, Baiyun, Guangzhou, 510540, China
}

(Received 21 September 2020; accepted 9 February 2021; published 8 March 2021)

\begin{abstract}
Active plasma lenses (APLs) have been proved as an effective device for focusing charged particles. In this paper, we have theoretically studied the feasibility of using a discharged-capillary based high gradient APL to focus laser accelerated pulsed proton beams. We specify equations of motion of proton beams in both straight and curved discharge-capillary APLs, on which the regulation law of a specific APL focusing and tuning protons can be calculated. Then, we summarize several constraints of APLs. Confirmed by a 2D PIC simulation, we find that with a proper plasma state, the proton energy loss, the transverse current distribution, and the Z-pinch effect do not significantly affect the beam transport, while the passive plasma lens (PPL) effect can promote the APL focusing within an acceptable extent. The regime of working parameters, including the plasma density $n_{0}$, the discharge current $I$, the length $L$ and the radius $R$ of APLs are given for different aimed proton energy. The results show that APL, whose parameters can be adjusted flexibly, is suitable for focusing and transporting proton beams with energy of 1-100 MeV. It is expected to build a beam-line based on plasma with centimeter length scale for pulsed proton beams. Such compact beam-line can have significant impact on various applications.
\end{abstract}

DOI: 10.1103/PhysRevAccelBeams.24.031301

\section{INTRODUCTION}

Particle acceleration based on high intensity laser systems (a process known as laser-plasma acceleration, LPA) has achieved high quality proton beams $[1,2]$. Such proton beams are accelerated after the formation of electron sheath [3] by means of spatially localized and temporally synchronized fields from relativistic laser plasma interactions [4], and exhibit exceptional beam properties including low emittance $\left(\sim 10^{-3} \mathrm{~mm} \cdot \mathrm{mrad}\right)$ [5], high intensity $\left(\sim 10^{13}\right.$ particles) and short pulse duration $(\sim \mathrm{ps})[6]$. Target normal sheath acceleration (TNSA) [7], the acceleration mechanism studied most extensively, has matured to a high level of robustness and reliability. This has made studies covering a wide range of laser and target parameters from many different experiments [8,9]. As a more efficient approach, radiation pressure acceleration (RPA), using

\footnotetext{
*1c0812@pku.edu.cn
}

Published by the American Physical Society under the terms of the Creative Commons Attribution 4.0 International license. Further distribution of this work must maintain attribution to the author(s) and the published article's title, journal citation, and DOI. the high radiation pressure of lasers to accelerate protons directly has also been explored [10]. So far, proton energies of up to $85 \mathrm{MeV}$ with TNSA [11] and $94 \mathrm{MeV}$ with hybrid RPA-TNSA mechanism [12] were generated using petawatt lasers with pulse duration $<1 \mathrm{ps}$ at ultrarelativistic light intensities of $10^{20} \mathrm{~W} / \mathrm{cm}^{2}$. The proton beams have a broad range of prospective applications such as material irradiation [13], imaging technology [14], cancer treatment [15], fast ignition [16], and warm-dense-matter generation [17]. For these applications, collimation and focusing of proton beams to keep the desirable properties (high peak current [18], micrometer source size [19], and low emittance [5]) is important.

During preliminary attempts to implement the application of the LPA protons, magnet quadrupoles [20], solenoids [21], and laser-triggered micro-lenses [22] are demonstrated as focusing elements. The quadrupole has to be used in a combination of triplet or doublet, because it cannot focus the beam symmetrically in two directions. Although the magnetic gradient of one quadrupole can reach up to $500 \mathrm{~T} / \mathrm{m}$, the effective acceptance angle of triplet or doublet quadrupoles is small due to the inevitable beam scattering and loss caused by the anisotropic focusing. A pulsed solenoid with $\mathrm{cm}$ inner diameter and 
magnetic strength of $2 \mathrm{~T}$ behind the target can catch most of the protons and symmetrically focus them into a monoenergetic beam, but it requires advanced pulsed magnet or superconducting technology. Microlenses, generated by a second short high-intensity laser pulse irradiating a tiny cylinder, can focus traversing protons due to the rapidly varying electric field. However, it suffers from complicated geometries and strict synchronization between the two lasers. Subsequently, various beam lines have been reported by several institutes, including the beam line at GSI Helmholtz Center, $[23,24]$ the ELIMED beam line installed in Prague for therapeutic purposes, [25] a compact gantry design constructed for LPA proton radiotherapy combined by pulsed magnets [26]. The CLAPA beam line at Peking University, which transports proton beams of high current with $1 \%$ energy spread reliably and practically [27]. The beam lines above based on traditional magnet combination are generally several meters long, which is unfavorable for miniaturization of laser plasma accelerators. They also encounter challenges when dealing with particles with broadband energy spectrum, owing to their sensitive dependence on energy [28]. The high peak current of the LPA protons is also an unsolved trouble because the space-charge field can not be neglected at micrometer size [29]. The generalized perveance [30] (an important parameter describing focus characteristics of beams) contributed by the space-charge fields is far larger than that of state-ofthe-art solenoids and quadrupoles. Plasma, as a macroscopic neutral medium composed of free electrons and ions, can effectively neutralize the pulsed beam, strongly relax the space charge restrictions, and provide certain selffocusing of the beam, which is considered as the convenient medium for beam transport [31]. Plasma can also sustain extremely large fields [32] and currents [33]. When a strong discharge current moving in the direction of the beam and passing through the plasma simultaneously, according to the Ampere law, an azimuthal magnetic field is generated, which provides symmetric focusing of particles and preserves the beam with longitudinal phase space [34]. This current-based magnetic lens with focused magnetic gradient up to $\mathrm{kT} / \mathrm{m}$ is called active plasma lens (APL). Distinguished from the passive plasma lens (PPL) [35] that relies on the self-field driven by the beam in plasma, APL is driven by an active, external current. The field of a PPL is strongly affected by the beam parameters (charge, current profile, and size), which limits its tunability. The field of an APL is generated by discharged current and can be flexibly adjusted, representing an alternative approach of new compact devices.

The first APL with only $4 \mathrm{ft}$. length was constructed to focus $350 \mathrm{MeV}$ proton beams by Panofsky and Baker in 1950 at BNL [36]. Later, APLs have been installed on ions beam lines at CERN [37] and GSI [38], implementing symmetrical focus of different kinds of ions, such as from $\mathrm{MeV}$ Krypton ions to GeV Gold ions. Remarkable precise focusing properties of APLs over traditional magnetic elements have been demonstrated [39]. However, the radii of the APLs above are over centimeter level. So high discharge current induces z-pinch effect and causes additional instability, thus limiting the application of APLs [40].

In recent years, APLs based on gas-filled discharge capillaries [41] have been found, which can produce highmagnetic gradients with flexible tunability. Gas-filled discharge capillaries are typically described as a cm-length gas-filled elongated volume with $\mathrm{mm}$-diameter circular cross section embedded in a glass or sapphire block, in which the plasma is produced by an electrical discharge current between two electrodes at both ends. The capillary plasma can effectively guide relativistic intense laser pulse, resulting in the generation of quasimonoenergetic electron beams with the highest energy up to $7.8 \mathrm{GeV}$ [42]. A curved plasma channel in a capillary has also been achieved experimentally [43]. It is proved that the accelerated electrons moving along the trajectory in a curve capillary can produce synchrotron radiation [44], which becomes feasible for both compact multiple acceleration stages [45] and synchrotron radiation source [46]. The peak current in the capillary can easily reach dozens of kA because the duration of the discharge pulse is only hundreds of nanoseconds, corresponding to a magnetic gradient higher than $3000 \mathrm{~T} / \mathrm{m}$. So attentions have also been paid to the focusing and guiding for charged particles through the channel. With proper synchronization between the current and the LPA electrons, several experiments performed in LBNL, [28] INFN [47] and Oxford [48] have reported radially symmetrical focusing of electrons successfully with the capillary discharge APLs, indicating their potential for compact laser accelerator applications.

Unlike LPA electron beams, LPA proton beams are nonrelativistic and much more divergent. Besides, considering the larger mass of protons, more stringent experimental conditions are required, compared to electrons. The goal of this paper is to investigate a solution to focusing and guiding the LPA pulsed proton beam by using a discharge capillary plasma. First, we analytically specify that both straight and curved discharge-capillary APL can focus the proton beam effectively in Sec. II. Then, we analyze the physical constraints of proton propagation in plasma channels in Sec. III and verify them in a 2-dimensional particle-in-cell (2D PIC) simulation. The method to meet the constraints is reported in Sec. IV. The possible challenges in the experiment are discussed in Sec. V. A conclusion is given in Sec. VI.

\section{ACTIVE PLASMA LENSES}

\section{A. Straight plasma channel APL}

An ideal APL, assuming a cylindrical channel filled with plasma of uniform conductivity, can provide radially symmetrically uniform focusing field. Figure 1(a) illustrates 


\section{B. Curved plasma channel APL}

(a)

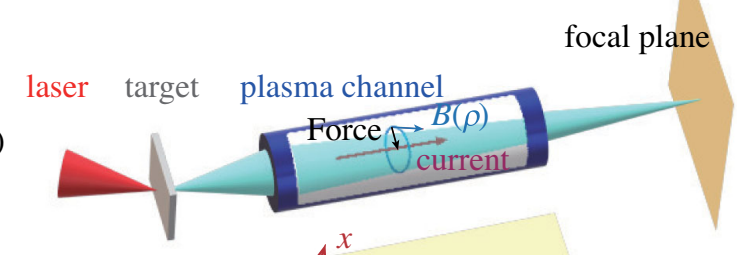

(b)

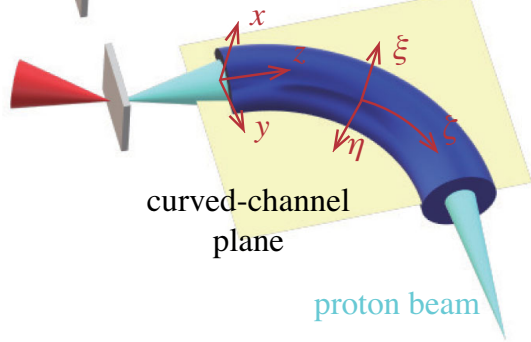

FIG. 1. Schematic concept of a proton beam focusing propagating in a straight (a) and a curved (b) APL. In the case of the curved APL, the coordinate system is transformed from the Cartesian coordinate system to the orthogonal toroidal one $(\xi, \eta, \zeta)$ as shown in (b).

the principle of the APL: a proton beam collinearly propagating along an actively externally current $I$ is radially focused. According to Ampere's law the radial magnetic gradient of a current with uniform density is

$$
\left(\frac{\partial B}{\partial \rho}\right)_{\text {uniform }}=\frac{\mu_{0} I}{2 \pi r^{2}}
$$

where $B$ is the azimuthal magnetic strength, $\rho$ is the radial distance from the axis of channel, $\mu_{0}$ is the vacuum permeability and $r$ is the radius of the circular capillary. Assuming $I=1000 \mathrm{~A}$ and $r=200 \mu \mathrm{m} \sim 500 \mu \mathrm{m}$, the corresponding magnetic gradient $\partial B / \partial \rho$ can reach 1000-5000 T/m.

Under the effect of a constant radial magnetic gradient, protons make a linear betatron motion [49] which can be described by Hill's equation

$$
\frac{d^{2} \rho}{d z^{2}}+K \rho=0
$$

where $K=q(\partial B / \partial \rho) /\left(m_{0} \gamma \beta c\right)$ is the focusing function of an ideal APL, $q$ is the proton charge, $m_{0}$ is the proton rest mass, $c$ is the vacuum speed of light and $\gamma$ and $\beta$ are the Lorentz factors. For an APL with a length of $L$, the equation above characterizes the focusing effect with a focal length

$$
f=\frac{1}{\sqrt{K} \sin \sqrt{K} L}
$$

Due to the high-magnetic gradient, $f$ is of the same order of $L$, because of which we will not adopt the thin-lens approximation in the following calculation.
Proton propagation in a curved plasma channel with uniform current can also be analyzed by orthogonal toroidal coordinates $(\xi, \eta, \zeta)$ shown in Fig. 1(b), where $\zeta$ is the distance along the curved channel axis and $\rho=$ $\left(\xi^{2}+\eta^{2}\right)^{1 / 2}$ is defined with respect to the channel axis. The transform from $(x, y, z)$ to $(\xi, \eta, \zeta)$ is [50]

$$
\left\{\begin{array}{l}
\xi=x \\
\eta=R-\sqrt{(R-y)^{2}+z^{2}} \\
\zeta= \begin{cases}R \arccos \frac{R-y}{\sqrt{(R-y)^{2}+z^{2}}}, & z \geq 0 \\
R\left(2 \pi-\arccos \frac{R-y}{\sqrt{(R-y)^{2}+z^{2}}}\right), & z \leq 0\end{cases}
\end{array}\right.
$$

Considering a channel which is curved in the $(y, z)$ plane with a constant radius of curvature $R$, we rewrite transverse components of Newton's second law $m d^{2} \vec{\rho} / d t^{2}=q \vec{v} \times \vec{B}$ with toroidal coordinates based on Jacobian determinant as

$$
\left\{\begin{array}{l}
\frac{d^{2} \xi}{d \zeta^{2}}+K \xi=0 \\
\frac{d^{2} H}{d \zeta^{2}}+K H=0
\end{array}\right.
$$

where $H=\eta+\eta_{0}$ is the distance form the balance point, $\eta_{0}=1 /(K R)$ is the distance between the balance point and the curved channel axis along the direction of $R$. The equations above have the same form as Hill's equation (2), which indicates that a curved plasma channel can guide and focus charged particles simultaneously, and the following analysis method is applicable to both straight and curved lenses.

\section{Focusing and tuning of APL on protons}

The effective focal length $F$ is the most concerned parameter of a magnetic focusing element. The parameter of a radially symmetric solenoid lens and an APL is $F=$ $\left(2 m_{0} \gamma \beta c / q\right)^{2} /\left(B^{2} L\right)$ and $F=f$, respectively. For a quadrupole, the expression $F= \pm f$ represents the effective focal length in both orthogonal directions. The optimized ideal triplet configuration $\pm(2 f,-f, f)$ can achieve radially symmetrical focusing, where each quadrupole is separated by distance $s=f_{0}$ and $f_{0}$ is the single-element focal length at the specific design energy $(50 \mathrm{MeV}$ in Fig. 2), yielding $F=2 s+\left(2 f^{3}-f s^{2}\right) /\left(f^{2}+f s-s^{2}\right)$. Note that the Lorentz factors $\gamma(\approx 1$ for multi-MeV protons $)$ and $\beta(\approx 1$ for relativistic electrons) in the expressions of $F$ have the same weight for all focusing elements, and therefore the scaling laws of the focusing strength with $\gamma$ for relativistic electrons proposed by Ref. [28] also applies to that with $\beta$ for multi-MeV protons. Figure 2(a) displaces the chromatic dependence $|\Delta \mathrm{F}|$ for a solenoid (black line), a quadrupole triplet (blue line) and an APL (red line) 


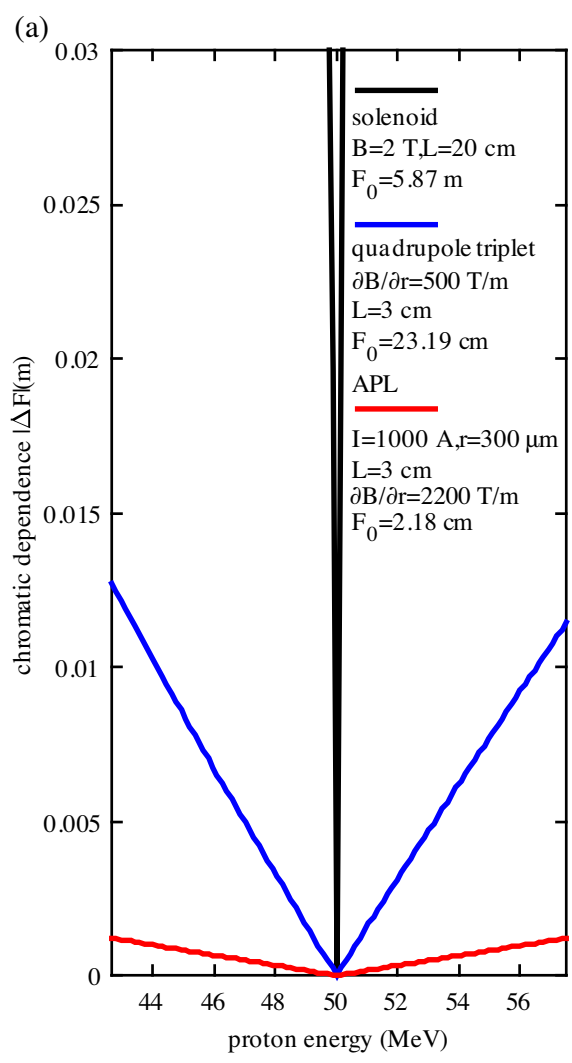

(b)

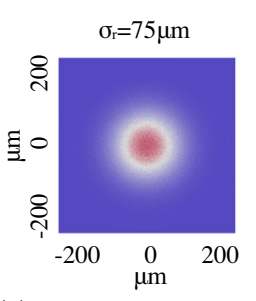

(c)

quadrupole triplet

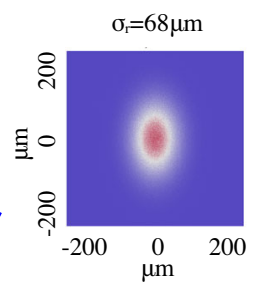

(d)

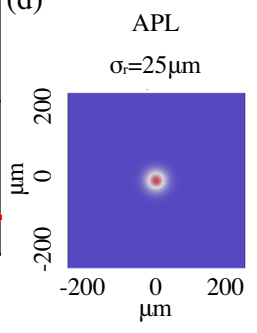

FIG. 2. (a) The chromatic dependence $|\Delta \mathrm{F}|$ is displayed for a solenoid (black line), a quadrupole triplet (blue line) and an APL (red line) designed for $50 \mathrm{MeV}$ protons. The focal spot of an initial collimated proton beam with energy spread of $30 \%$ around $50 \mathrm{MeV}$ is displayed on the right side, after focused by the solenoid (b), the quadrupole triplet (c) and the APL (d) with the marked parameters values, respectively. The rms transverse radius of the initial beam with a bi-Gaussian distribution is $300 \mu \mathrm{m}$.

designed for $50 \mathrm{MeV}$ protons, with focal lengths $F=5.87 \mathrm{~m}, 23.19 \mathrm{~cm}$ and $2.18 \mathrm{~cm}$, respectively. The corresponding proton spots on the focal plane are also shown in Fig. 2 from (b) to (d), for a collimated initial incident proton beam with a rms spot transverse radius $\sigma_{r 0}=300 \mu \mathrm{m}$ (bi-Gaussian distribution) and an energy spread of $30 \%$ around $50 \mathrm{MeV}$. One can observe that the APL has the shortest focal length, the weakest energy dependence and the smallest beam focal spot.

The adjustability of a focusing element is also an important parameter in order to meet the complex and changeable requirements in the experiments. We define the length of a capillary APL as $L$, the distance between the proton source (object point) and the entrance of the capillary as object distance $l$, the distance between the exit of the capillary and the focused spot (image point) as image distance $d$, and the distance between the object point and the image point as $S(l+L+d=S)$. Figure 3 shows two different adjustment methods for a APL with $L=3 \mathrm{~cm}$ and $r=300 \mu \mathrm{m}$. First, assuming that $l=4 \mathrm{~mm}$ is fixed in the
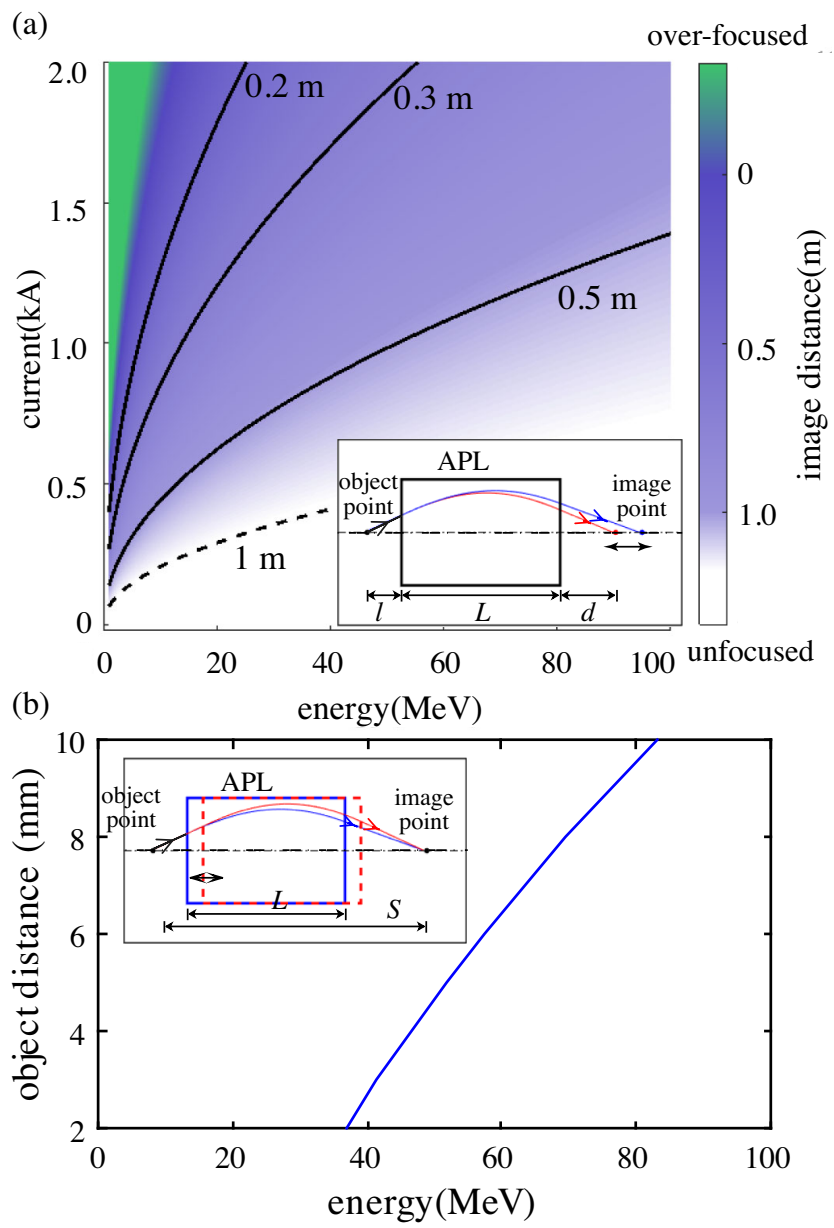

FIG. 3. Two different adjustment methods for an APL with $L=$ $3 \mathrm{~cm}$ and $r=300 \mu \mathrm{m}$. The parameters involved in the figure are APL length $L$, object distance $l$ (the distance between the object point and the entrance of APL), image distance $d$ (the distance between the image point and the exit of APL) and distance between object and image point $S$. (a) Scaling of the image distance $d$ with discharged current and aimed beam energy. The object distance is fixed at $l=4 \mathrm{~mm}$. The black dotted line is the lower boundary of $I$ demarcated from unfocused beams, where $d=1 \mathrm{~m}$. (b) Scaling of the object distance with the aimed proton energy when the distance between the object and image point is fixed at $S=0.5 \mathrm{~m}$ and the discharge current is also fixed at $I=1 \mathrm{kA}$. The inset subgraphs show two different adjustment methods corresponding to Fig. 3(a) and (b), respectively.

experiment, we try to find the optimum capillary discharge current to achieve the smallest beam spot at an adjustable image point for protons with various energies. In Fig. 3(a), the blue area shows the scaling of the discharge current with the aimed proton energy for different $d$. For example, when $I=1 \mathrm{kA}$, the $50 \mathrm{MeV}$ protons can be focused at $d=0.5 \mathrm{~m}$. The black dotted curve in Fig. 3(a) is for $d=1 \mathrm{~m}$, which is set as the lower boundary of $I$, because a too large $d$ is not applicable for a compact device. While the green area in the left indicates that too high $I$ causes the protons over-focused inside the capillary and $d$ is negative. 
Generally, the position of the proton source and the irradiation point are fixed, meaning that $S$ is fixed in experiments. To focus aimed protons with various energies complying waist-waist transport in the case of the fixed $S$, we can either adjust the discharge current to keep $K$ constant along black lines shown in Fig. 3(a), or adjust the distance $l$. Fig. 3(b) shows the focusing regulation law for the capillary with same parameters in Fig. 3(a), and here $S=0.5 \mathrm{~m}$ and $I=1 \mathrm{kA}$. If the energy of the focused proton beams increases from $40 \mathrm{MeV}$ to $80 \mathrm{MeV}, l$ increases correspondingly from $2 \mathrm{~mm}$ to $10 \mathrm{~mm}$. Due to the small size of APLs, electric stepping motors can be used to adjust the posture of APLs with micrometer resolution. The combination of the two as a zoom lens can raise tunability in experiments, which is difficult to achieve with conventional magnetic transport elements. More adjustment methods in multidimension can be achieved for a curved APL. For instance, by rotating the exit angle of the capillary, we can easily adjust the propagation direction of the proton beam, which has the similar function of the curved AG-CCT magnet [51].

\section{PARAMETER LIMITATION ON APL}

The interaction of charged particles with plasma has important influences on focusing. The APL can effectively focus proton beams in a state where the plasma medium cannot significantly affect the intrinsic characteristics of the beam, such as emittance and energy.

\section{A. Proton energy loss}

Energy loss is a major aspect attracting attention in the focusing process. The incident proton beam transfers part of the energy to electrons and ions in fully ionized plasma with a rate much higher than that in solid [52]. Extensive research works indicate that the energy loss can be expounded by linear theories include the dielectric theory and the binary collision one, when the ion-plasma coupling strength $Z=Z_{p} / N_{D} \ll 1$, where $Z_{p}=1$ is the proton charge state, $N_{D}=n_{0} \lambda_{D}^{3}$ is the number of particles in the Debye sphere and $\lambda_{D}=\sqrt{\varepsilon_{0} k_{b} T_{e} /\left(n_{0} e^{2}\right)}$ is the Debye length [53]. This applies for high energy injection protons $\left(v_{p} \gg v_{\text {th }}\right)$ [52] and nonmagnetized $\left(\omega_{\mathrm{ce}} \ll \omega_{\mathrm{pe}}\right)$ [54] plasma. Here $v_{p}=\sqrt{2 E / m_{0}}$ is the proton velocity, $v_{\text {th }}=\sqrt{3 k_{b} T_{e} / m_{e}}$ is the plasma electron thermal velocity, $\omega_{\mathrm{ce}}=e B / m_{e}$ is the electron cyclotron frequency, $\omega_{\mathrm{pe}}=\sqrt{n_{0} e^{2} /\left(\varepsilon_{0} m_{e}\right)}$ is the plasma frequency, $E$ is the proton energy, $k_{b}$ is the Boltzmann constant, $T_{e}$ is the plasma temperature, $m_{e}$ is the electron mass, $e$ is the electronic charge, $n_{0}$ is the plasma density and $\varepsilon_{0}$ is the vacuum permittivity. For proton beams with energy higher than a few $\mathrm{MeV}$ and discharged plasma with $T_{e}$ on a level of several $\mathrm{eV}, n_{0}$ below $10^{17} \mathrm{~cm}^{-3}$ and $B \sim 1 \mathrm{~T}$, the linear theory is well satisfied. The expression of the proton energy loss can be written as a modified Bethe formula [55]

$-\left(\frac{d E}{d z}\right)=\frac{Z_{p}^{2} e^{2} \omega_{\mathrm{pe}}^{2}}{4 \pi \varepsilon_{0} v_{p}^{2}} G\left(\frac{v_{p}}{v_{\mathrm{th}}}\right) \ln \left(\frac{4 \pi \varepsilon_{0} m_{e} v_{p}^{3}}{Z_{p} e^{2} \omega_{\mathrm{pe}}} L_{\mathrm{Bloch}}\right)$

where $G\left(v_{p} / v_{\text {th }}\right)$ is the Chandrasekar function [56] and $L_{\text {Bloch }}$ is the Bloch modified term [57]. The calculation result is $(d E / d z) \sim 0.01 \mathrm{MeV} / \mathrm{m}$ for the condition above, representing the energy loss of $\mathrm{MeV}$ protons propagating in a capillary plasma can be neglected.

\section{B. Quasineutrality condition}

When propagating in the plasma, the proton beam carries an equal number of electrons, therefore together they can be described as moving plasma which meets the quasineutrality condition. There is an upper limit on the charge density of protons inside the local plasma that can be focused by the magnetic field of the APL, because the condition that the magnetic skin depth is much longer than the beam radius must be met [58], that is

$$
\frac{c}{\omega_{p b}} \gg \frac{\sigma_{r}}{2}
$$

where $\omega_{p b}=\sqrt{n_{b} e^{2} /\left(\varepsilon_{0} m_{e}\right)}$ is the local plasma frequency, $n_{b}=N /\left[(2 \pi)^{3 / 2} \sigma_{z} \sigma_{r}^{2}\right]$ is the proton beam density assuming a bi-Gaussian distribution, $\sigma_{r}$ is the rms transverse beam size, and $\sigma_{z}$ is the rms beam length. The expression indicates the upper limit on number of protons $N \ll\left(c^{2} m_{e} \varepsilon_{0} \sigma_{z}\right) / e^{2}$. For the beam parameter $\sigma_{z}=30 \mu \mathrm{m}$, the limit is about $10^{9}$.

\section{Transverse current distribution}

The analysis above is based on the assumption that the discharge current is uniform, however, magnetohydrodynamics (MHD) simulations show that the transverse current distribution in the plasma lens is nonuniform [59]. From the $J \sim T_{e}^{3 / 2}$ model with $J$ the current density [60], we calculate the $B$ scaling with the radial distance from the central axis of the capillary $\rho$, as shown in Fig. 4(a). Here, we still assume a capillary APL with $I=1 \mathrm{kA}$ and $r=300 \mu \mathrm{m}$. One can observe an enhancement in magnetic gradient for the nonlinear model compared with the uniform model. Reference [61] gives an analytical approach to optical properties with a nonlinear magnetic field profile. Figure 4(b) shows the scaling of the beam emittance increase $\Delta \varepsilon$ and the actual focal length $f_{a c}$ with the spot radius of the incident beam in the case of the nonuniform current. For a collimated proton beam with energy of $50 \mathrm{MeV}$, the analytic focal length of the APL in the case of uniform current is $2.18 \mathrm{~cm}$. We can see that $f_{a c}$ is shorter than that of the uniform current case and the potential emittance deterioration is severer at larger beam radius. The primary consequence of the $B$ nonlinearity is the reduction 


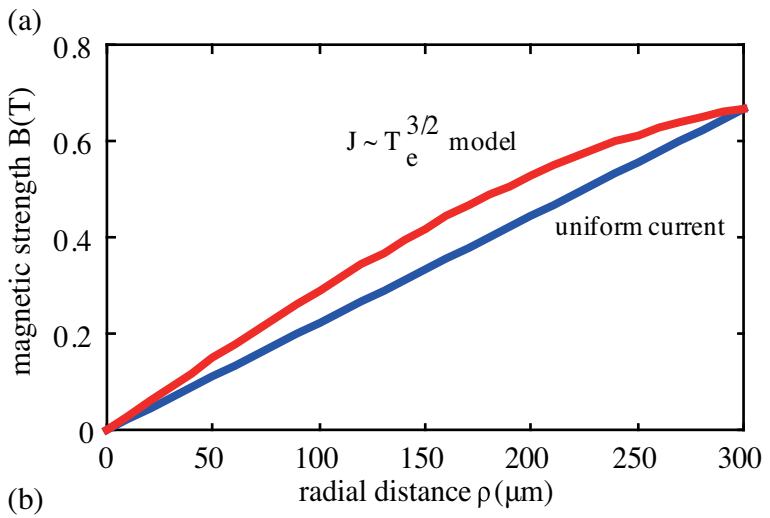

(b)

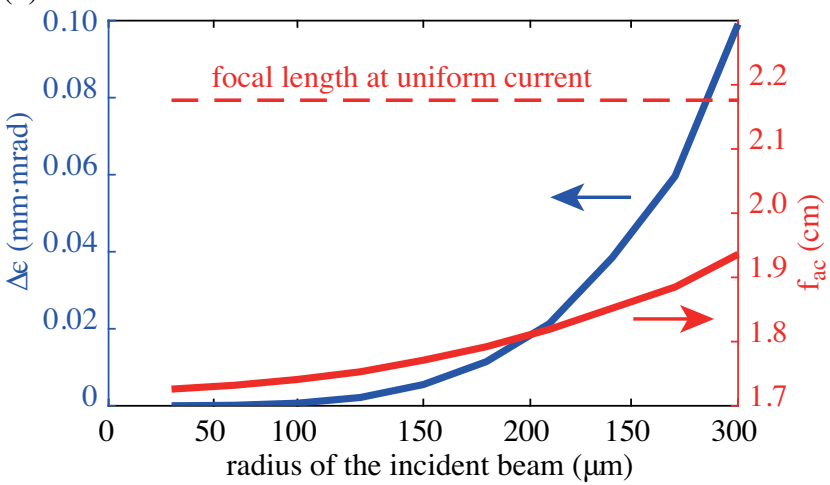

FIG. 4. (a) The magnetic field profile calculated by the model based on $J \propto T_{e}^{3 / 2}$ (red curve) and by the uniform current assumption (blue curve) for a capillary with radius $r=300 \mu \mathrm{m}$ and discharge current $I=1 \mathrm{kA}$. (b) The scaling of emittance increase $\Delta \varepsilon$ (blue curve) and actual focal length $f_{\text {ac }}$ (red curve) with incident spot size of an ideal parallel beam in the case of the magnetic strength distribution shown by the red curve in (a).

of the effective APL radius from $300 \mu \mathrm{m}$ to about $200 \mu \mathrm{m}$, which is consistent with the conclusion of Ref. [62] that particles outside $r / 3$ in the APL do not contribute efficiently to the high-quality focus. One solution to suppressing the $B$ nonlinearity is to use heavier species of gas to discharge [48]. The effective plasma channel radius can be maintained due to current uniformity improved by more radiation heating.

\section{Z-pinch effect}

Another limiting factor is the z-pinch effect [40] that reduces the plasma channel radius and induces plasma instabilities, leading to degradation of beam quality. In particular, the contraction of effective plasma radius should be avoided to protect the collection angle. The pinch effect can be ignored if the plasma pressure $p_{T}$ is larger than the magnetic pressure $p_{B}$, which means

$$
n_{0} k_{b} T_{e}>\frac{\mu_{0} I^{2}}{8 \pi^{2} r^{2}}
$$

The condition demonstrates that there is an upper limit for the focusing strength under given $n_{0}$ and $r$, which means the focal length $f$ has a minimum value. For the parameters above $\left(n_{0} \sim 10^{16}-10^{18} \mathrm{~cm}^{-3}, \quad T_{e} \sim \mathrm{eV}\right.$, $I \sim \mathrm{kA}$ ), the Inequality (8) is well satisfied and the plasma channel radius is almost equal to the capillary radius.

\section{E. PPL effect}

Passive plasma lens effect is always present even in an APL [63]. When the beam of positively charged particles moves through the plasma, the plasma electrons bring two effects on the beam: neutralization of the beam charge and neutralization of the beam longitudinal current [64]. For a beam with a radius $\sigma_{r}<c / \omega_{\mathrm{pe}}$, the longitudinal current is not completely neutralized [65]. So it will experience the self-focusing due to the shielding process produced when the background plasma reorganizes to conserve the overall neutrality. At the same time, a comoving plasma wakefield is generated by the disturbance of the protons to the background electrons, [66] and the profile of the beam would be modulated by the radial wakefield. The inclusion of nonlinear passive plasma wakefield on the beam also affects the beam emittance [67]. However, it is hard to give an analytical description of the PPL effect for a nonrelativistic proton beam with broadband energy spectrum.

In order to evaluate the related impacts, we have performed 2D PIC simulations with EPOCH [68]. The simulated transport domain is $300 \mu \mathrm{m} \times 3 \mathrm{~cm}$ in the $y-z$ plane. The simulations consider a uniform density plasma channel with density $n_{0}=10^{16} \mathrm{~cm}^{-3}$ located at $0<z<2 \mathrm{~cm}$. Initially $t=0$, a laser accelerated proton beam with an exponential decaying energy spectrum from 8.5 MeV to $11.5 \mathrm{MeV}$ and a Gaussian density profile $n(y, z)=n_{b} \exp \left(-y^{2} / 2 \sigma_{r}-\ln 10 z / \sigma_{z}\right)$, where $n_{b}=10^{16} \mathrm{~cm}^{-3}$ and $\sigma_{z}=30 \mu \mathrm{m}$, is located at $z$ origin as channel entrance. Considering a few $\mathrm{mm}$ drifting before entering the APL, the proton energy $E$ establishes a linear chirp in $z$ direction, i.e., $E(z)[\mathrm{MeV}]=8.5+3 z / \sigma_{z}$. To save computing resources, we adapted moving-window method. As $\sigma_{z}$ increase along with transmission, the domain of the window is $300 \mu \mathrm{m} \times 6 \mathrm{~mm}$ with $600 \times 12000$ cells moving from $z=0$ to $z=3 \mathrm{~cm}$ at the same speed as the highest energy (11.5 MeV) proton. A uniform linear magnetic field $B(y)=k y$ perpendicular to simulation plane is added in plasma domain, where $k=$ $1837 \mathrm{~T} / \mathrm{m}$ is magnetic gradient. The focus image point is expected at $z=2.5 \mathrm{~cm}$ for $10 \mathrm{MeV}$ protons using Eq. (3).

The simulation results after analysis is given in Fig. 5. Figure 5(a) shows the evolution of the transverse beam density profile with $z$ from the initial $z=0$ to different detection planes $z=2.3 \mathrm{~cm}, 2.5 \mathrm{~cm}$ and $3 \mathrm{~cm}$. In the experiment, we usually use a truncated beam profile detector, such as a scintillator, to measure the accumulative superposition of the energy of all protons in a beam. Therefore, the beam density profiles in Fig. 5(a) are also the superposition of the whole beam with the broad energy spectrum. We define the focal plane at the position where 

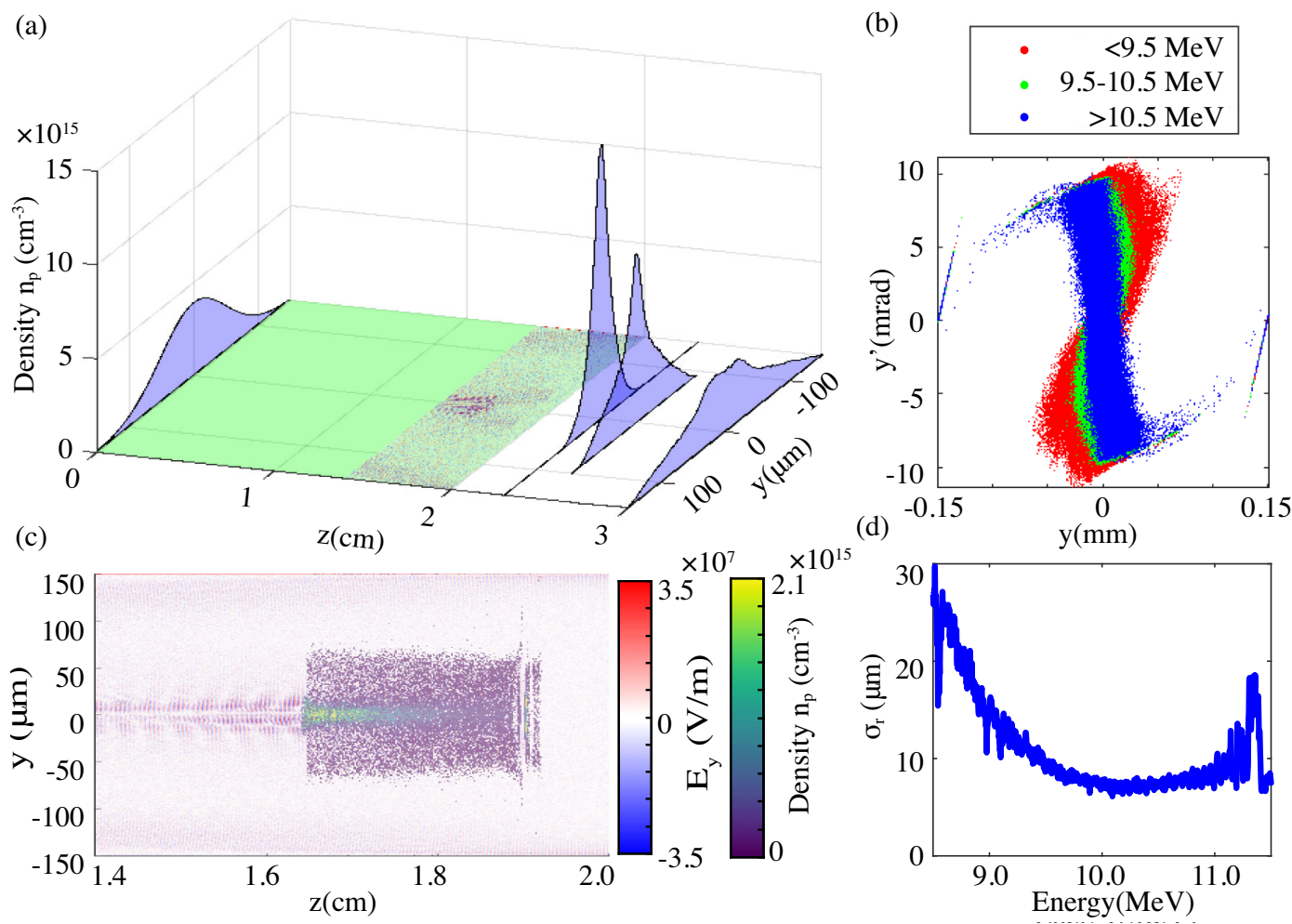

FIG. 5. The 2D PIC simulation for a proton beam with a broad energy spectrum focused by an APL located from $z=0$ to $2 \mathrm{~cm}$. (a) Scaling of the density profiles of the entire beam at different $z$, from the initial $z=0$ (before entering the APL) to $z=2.3,2.5$, and $3 \mathrm{~cm}$ (different detection planes behind the APL). The waist of the beam is at focal plane $z=2.3 \mathrm{~cm}$. (b) The density distribution of the proton beam shown in the moving window when it propagating close to the exit of the APL at $z=1.9 \mathrm{~mm}$. The electric field caused by the background electron disturbance (wakefield) is also superimposed. (c) Phase diagram $y^{\prime}-y$ of the proton beam at $z=2.3 \mathrm{~cm}$. Protons with $10 \%$ energy spread around $11 \mathrm{MeV}, 10 \mathrm{MeV}$ and $9 \mathrm{MeV}$ are marked with blue, green, and red colors. (d) Scaling of the rms beam radius $\sigma_{r}$ with the protons energy at $z=2.3 \mathrm{~cm}$.

the beam can be focused to a waist $\sigma_{r}=16.6 \mu \mathrm{m}$, that is $z=2.3 \mathrm{~cm}$ here. Compared with the result of the matrix calculation $(z=2.5 \mathrm{~cm}$ for $10 \mathrm{MeV})$, the focal length obtained by the simulation is $8 \%$ shorter, indicating that the self-focusing caused by the PPL effect [63] improves the overall focusing ability for the beam. For a plasma medium, certain parameters are approximate whether in calculation or simulation, and the accurate $K$ needs to be revised according to the experiment. But it is proved that APL effect is absolutely dominant compared with PPL effect, so we can still use the theoretical calculation result as a prediction of experiments.

During the propagation, the beam stretches longitudinally as well due to the large energy chirp. Figure 5(b) shows the density profile in the moving window at $t=$ 408 ps when the entire beam is close to the exit of the APL plasma. It is focused transversely and stretches to millimeter longitudinally. The $E-y$ caused by the background electron density perturbation (wakefield) [66] is also superimposed, with wave length of $\lambda_{p}=2 \pi \beta c / \omega_{\mathrm{pe}} \sim 50 \mu \mathrm{m}$ [69], which is much shorter than that of the beam.
The nonlinear interaction with plasma may induce microstructures on the beam and deteriorate its transverse emittance [67], causing phase diagram distortion. Figure 5(c) is the phase diagram of the proton beam at the focal plane $z=2.3 \mathrm{~cm}$, where protons with $<9.5 \mathrm{MeV}, 9.5-10.5 \mathrm{MeV}$, and $>10.5 \mathrm{MeV}$ are marked with red, green, and blue colors. It shows that protons with higher energy are weakly affected by the plasma, but the wakefield produced by them will affect the subsequent lower-energy protons. From the phase diagram, the emittance of the entire beam with broadband energy spectrum [70] at focus is calculated as $\Delta \varepsilon=0.06 \mathrm{~mm} \cdot \mathrm{mrad}$, which is still small comparing with that of traditional accelerators $(\sim \mathrm{mm} \cdot \mathrm{mrad})$. Therefore, we consider that the influence by self-driven wakefield can be acceptable. In addition, increasing the plasma density and adopting the non-uniform plasma are the effective ways to restrain the wakefield [64]. Figure 5(d) shows scaling of rms beam radius $\sigma_{r}$ with proton energy at the focal plane. For beams with broad energy spread, the $\sigma_{r}$ undulates within $20 \mu \mathrm{m}$, indicating that focusing through APLs indeed weakly depends on energy. 


\section{APL PARAMETERS SEARCHING METHOD}

The working parameters of APL are limited by the multiple conditions above with implicit relationships. Based on the analysis above that PPL effect is relatively minor, we put forward a method to search the range of parameters at working state. To design an APL for a center proton energy $E$, four typical parameters $\left(L, r, I, n_{0}\right)$ need to be determined. Obviously, this is a nonlinear multivariable optimization [71] to be solved.

First, we write the energy loss constraint as $\int_{0}^{L}(d E / d z) d l \leq \nu E$, where $\nu$ is the acceptable rate of energy loss and $(d E / d z)$ is a function of $n_{0}$ and $L$. For a short-pulse proton beam propagating in plasma, $(d E / d z)$ should be obtained by PIC simulation rather than Eq. (6). However, the nonlinear inequality constraint in integral form will make the optimization too complicated. Following the analysis above in Sec. III A, if we make sure that the APL work in linear region, then this constraint can be ignored.

The constraint due to the z-pinch effect is shown by inequality (8), in which $T_{e}$ is also related to $n_{0}$ and $I$. Using the MHD simulation, people demonstrate that the temperature does not change sensitively with $n_{0}$ and $I$, so one can estimate $T_{e}$ as a constant temperature, for example, $5 \mathrm{eV}$ in most of the discharge parameter range. Under this assumption, inequality (8) can be simplified to a linear constraint with three parameters $\left(n_{0}, I\right.$, and $\left.r\right)$. We optimize the problem for various $n_{0}$ respectively.

Another constraint is that the beam coming out of the APL must be converged. The laser accelerated protons can be considered as emitting from a virtual point source [19]. If the distance from the source point to the entrance of the capillary APL is $l$, the angle $r^{\prime}$ of a proton passing through the lens at the exit of the APL can be written as

$$
r^{\prime}=-l r_{0}^{\prime} \sqrt{K} \sin \sqrt{K} L+r_{0}^{\prime} \cos \sqrt{K} L
$$

where $r_{0}^{\prime}$ is the initial angle of the proton. Only if $r^{\prime}<0$, the converging condition of the proton beam is met, which can be described as

$$
\sqrt{K} L \gtrsim \frac{\pi}{2}
$$

The acceptance angle $\theta$ of the APL for a beam from a point source can be described as

$$
\theta=\frac{r}{\sqrt{l^{2}+\frac{1}{K}}} .
$$

Only those protons with initial angle smaller than $\theta$ can pass through the APL. To enlarge $\theta$ and collect more protons, the distance $l$ should be small. We transform the angle formula and get

$$
\frac{1}{\theta^{2}}=\frac{l^{2}+\frac{1}{K}}{r^{2}}=\frac{l^{2}}{r^{2}}+\frac{2 m_{0} \pi \beta c}{q \mu_{0} I} .
$$

To optimize the problem for various $n_{0}$, we rewrite the parameters $(L, r, I)$ as independent variable $\left(x_{1}, x_{2}, x_{3}\right)$ and the objective function in form of fractional polynomial. The optimization is specified by

$$
\begin{aligned}
& \min _{x_{1}, x_{2}, x_{3}} \frac{l^{2}}{x_{2}^{2}}+\frac{2 m_{0} \pi \beta c}{q \mu_{0} x_{3}} \text { such that } \\
& \left\{\begin{array}{l}
\frac{\pi^{3}}{2} m_{0} \beta c x_{2}^{2}-q \mu_{0} x_{3} x_{1}^{2} \leq 0 \\
-\sqrt{\frac{8 \pi^{2}}{\mu_{0} n_{0} k_{b} T_{e}}} x_{2}+x_{3} \leq 0 \\
10 \leq x_{1} \leq 100,0.1 \leq x_{2} \leq 2
\end{array}\right.
\end{aligned}
$$

where the unit of length is $\mathrm{mm}$ in the bound constraints, in which the specific values are determined by experimental conditions.

Assuming $l=4 \mathrm{~mm}$, we implement optimization above for $1-100 \mathrm{MeV}$ proton energy under different plasma densities $\left(10^{16}-10^{18} \mathrm{~cm}^{-3}\right)$, and the results are shown in Fig. 6. Figure 6(a) indicates that the optimized maximum collection angle $\theta \downarrow$ with $E \uparrow$, for the reason $K \downarrow$ with $E \uparrow$; it also indicates that $\theta \uparrow$ with $n_{0} \uparrow$, for the reason $K \uparrow$ with $I \uparrow$. In the optimization, $I$ is the most influential parameter. Figure 6(b) shows that the upper limit of $I \uparrow$ with $n_{0} \uparrow$ due to the z-pinch effect described by inequality (8). At the same time, inequality (10) requires $K$ to be large enough, which requires ratio $I / r^{2} \uparrow$ with $E \uparrow$. Under these two premises, $I \downarrow$ and $r \downarrow$ with $E \uparrow$ in first and second power level synchronously, which are shown in Fig. 6(b) and (d) respectively, to maintain the maximum magnetic gradient. On the other hand, converging constraint in inequality (10) can be compensated by $L \uparrow$ as shown in Fig. 6(c). The parameters above must be within the limit of the conditions to ensure APLs working in a stable focusing transmission state, and only when all parameters take the limit values simultaneously, the APL can achieve the maximum collection angle $\theta$.

\section{DISCUSSION}

From Eq. (6) we can get that the energy loss of protons with energy over $\mathrm{MeV}$ is not sensitive to plasma density of range lower than $10^{20} \mathrm{~cm}^{-3}$, and thus increasing $n_{0}$ is an effective way to relax the limitation inequality (8). Consequently, the focusing strength of APLs can be enhanced by continuously increasing the current. If the focusing strength is kept, the lens can be designed with a larger aperture so that the collection angle will increase. We recommend a hundred-of$\mu$ m-radius APL with $10^{16}-10^{17} \mathrm{~cm}^{-3}$ density and several-kA current to focus the protons with $\mathrm{MeV}$ level energy accelerated by laser pulses with $10^{18-19} \mathrm{~W} / \mathrm{cm}^{2}$ intensity, and a several-millimeter-radius APL with $10^{18} \mathrm{~cm}^{-3}$ density and 


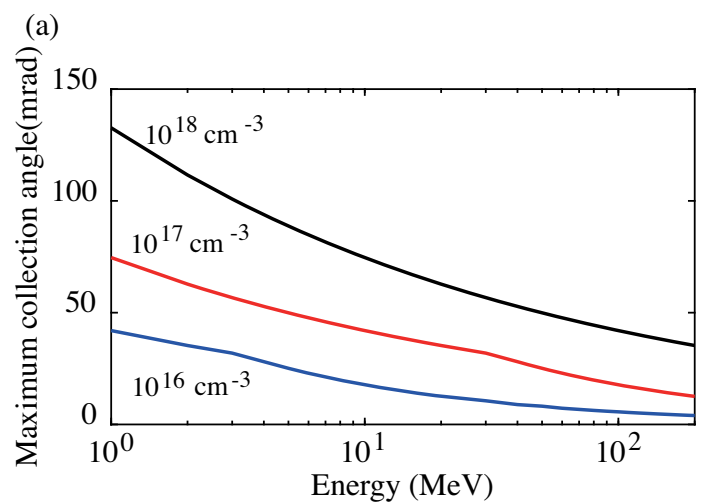

(b)

(c)
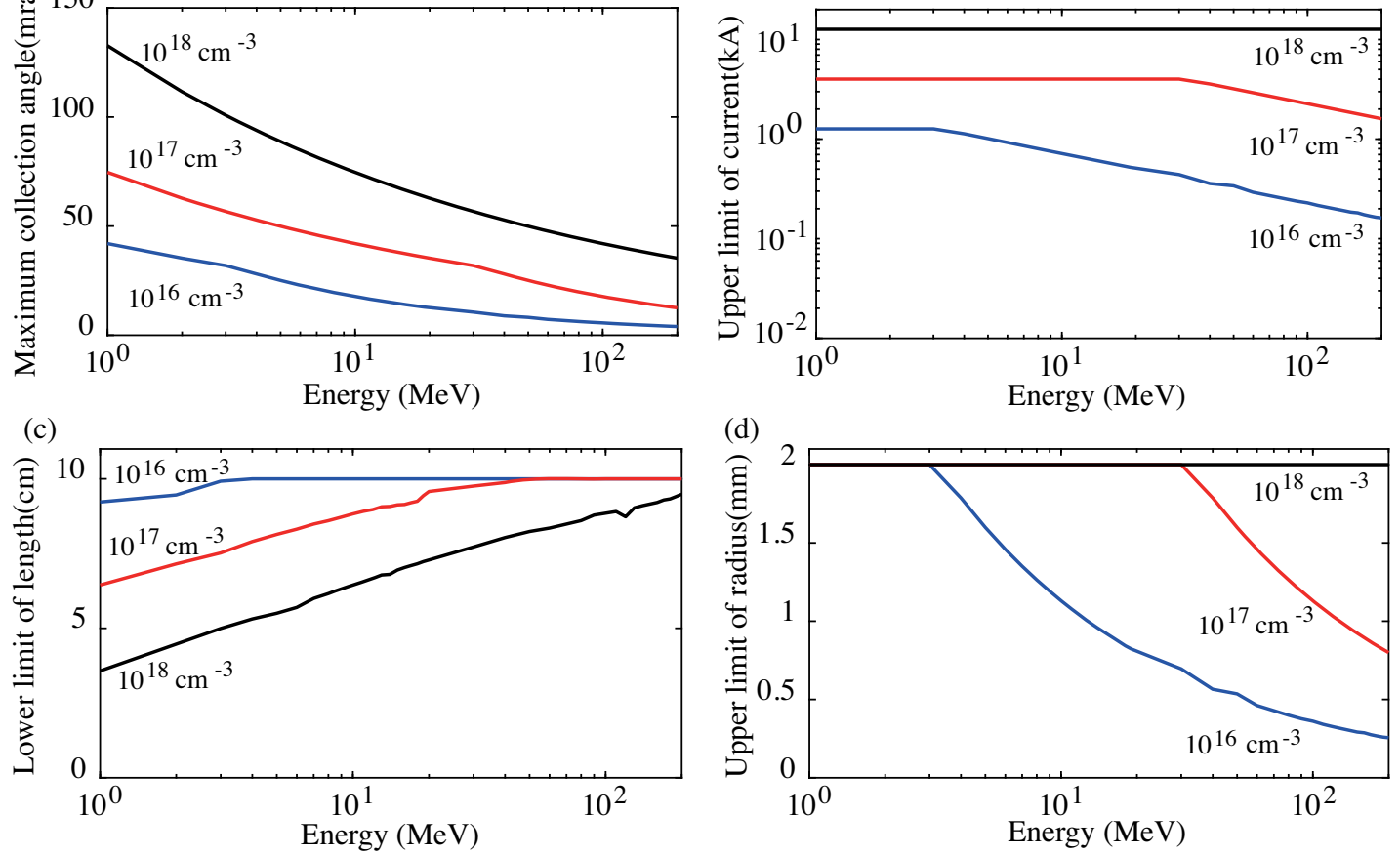

FIG. 6. The optimization result of the maximum collection angle (a), upper limit of current (b), lower limit of length (c) and upper limit of radius (d) for a working-state APL scaling with energy.

score-of-kA current to focus the protons with energy over scores of $\mathrm{MeV}$ accelerated by laser pulses with $10^{20-21} \mathrm{~W} / \mathrm{cm}^{2}$ intensity.

In experiments, the large divergence angle of LPA proton beams is a challenge to overcome for APLs based on discharge capillaries. The APL has to be placed close to the target in order to increase beam collection efficiency. At sufficiently small distances, protons can also enter APLs before the Coulomb explosion, thus maintaining a low initial emittance. However, there should also be a safe distance between the entrance of the APL and the target to avoid the damage of the micrometer thickness foil and the acceleration structure due to the ejected ionized gas from the capillary [72]. Changing the settings of the laser and target to decrease the initial divergence [73-75] is a solution to the problem. Or one can explore new technologies for mounting targets on the electrode.

\section{CONCLUSION}

In conclusion, we specify the discharge capillary APL is a feasible solution to focusing and guiding the LPA pulsed proton beam both in the straight and curved structure. We also demonstrate a systematic APL parameters searching method via a nonlinear multivariable optimization, dealing with many fundamental restrictions include the energy loss, the magnetic skin depth, the z-pinch effect and the wakefield. Via 2D PIC simulation we illustrate the unapparent impact of plasma on proton transport. The study confirms that the APL has one order of magnitude higher magnetic field gradient, shorter scale focal length and weaker energy dependence, compared with solenoids and quadrupoles. The hundred-of-ns discharge system of capillary APL is not only simple in setup, but also suitable for LPA proton beams with ultrashort pulse duration. The APL has the ability to tightly focus protons to a micrometer transverse size spot with good tunability, it also has great potential to minimize the beam line of laser accelerator, which have significant impact on various areas such as accelerator physics, inertial fusion science, astrophysics, or even proton oncology.

\section{ACKNOWLEDGMENTS}

This work was supported by the National Natural Science Foundation of China (Grants No. 11975037, No. 61631001, and No. 11921006), and the National Grand Instrument Project (No. 2019YFF01014400 and No. 2019YFF01014404).

[1] A. Maksimchuk, S. Gu, K. Flippo, D. Umstadter, and V. Y. Bychenkov, Forward Ion Acceleration in Thin Films Driven by a High-Intensity Laser, Phys. Rev. Lett. 84, 4108 (2000).

[2] A. Macchi, M. Borghesi, and M. Passoni, Ion acceleration by superintense laser-plasma interaction, Rev. Mod. Phys. 85, 751 (2013). 
[3] S. Steinke, J. H. Bin, J. Park, Q. Ji, K. Nakamura, A. J. Gonsalves, S. S. Bulanov, M. Thévenet, C. Toth, J.-L. Vay, C. B. Schroeder, C. G. R. Geddes, E. Esarey, T. Schenkel, and W. P. Leemans, Acceleration of high charge ion beams with achromatic divergence by petawatt laser pulses, Phys. Rev. Accel. Beams 23, 021302 (2020).

[4] M. Borghesi, J. Fuchs, S. V. Bulanov, A. J. MacKinnon, P. K. Patel, and M. Roth, Fast ion generation by highintensity laser irradiation of solid targets and applications, Fusion Sci. Technol. 49, 412 (2006).

[5] T. E. Cowan et al., Ultralow Emittance, Multi-Mev Proton Beams from a Laser Virtual-Cathode Plasma Accelerator, Phys. Rev. Lett. 92, 204801 (2004).

[6] B. Dromey, M. Coughlan, L. Senje, M. Taylor, S. Kuschel, B. Villagomez-Bernabe, R. Stefanuik, G. Nersisyan, L. Stella, J. Kohanoff, M. Borghesi, F. Currell, D. Riley, D. Jung, C. G. Wahlström, C. L.S. Lewis, and M. Zepf, Picosecond metrology of laser-driven proton bursts, Nat. Commun. 7, 10642 (2016).

[7] R. A. Snavely, M. H. Key, S. P. Hatchett, T. E. Cowan, M. Roth, T. W. Phillips, M. A. Stoyer, E. A. Henry, T. C. Sangster, M. S. Singh, S. C. Wilks, A. MacKinnon, A. Offenberger, D. M. Pennington, K. Yasuike, A. B. Langdon, B. F. Lasinski, J. Johnson, M. D. Perry, and E. M. Campbell, Intense High-Energy Proton Beams from Petawatt-Laser Irradiation of Solids, Phys. Rev. Lett. 85, 2945 (2000).

[8] H. Daido, M. Nishiuchi, and A. S. Pirozhkov, Review of laser-driven ion sources and their applications, Rep. Progr. Phys. 75, 056401 (2012).

[9] J. Schreiber, P. R. Bolton, and K. Parodi, Invited review article: "hands-on" laser-driven ion acceleration: A primer for laser-driven source development and potential applications, Rev. Sci. Instrum. 87, 071101 (2016).

[10] T. Esirkepov, M. Borghesi, S. V. Bulanov, G. Mourou, and T. Tajima, Highly Efficient Relativistic-Ion Generation in the Laser-Piston Regime, Phys. Rev. Lett. 92, 175003 (2004).

[11] F. Wagner, O. Deppert, C. Brabetz, P. Fiala, A. Kleinschmidt, P. Poth, V. A. Schanz, A. Tebartz, B. Zielbauer, M. Roth, T. Stöhlker, and V. Bagnoud, Maximum Proton Energy above $85 \mathrm{mev}$ from the Relativistic Interaction of Laser Pulses with Micrometer Thick $\mathrm{ch}_{2}$ Targets, Phys. Rev. Lett. 116, 205002 (2016).

[12] A. Higginson, R. J. Gray, M. King, R. J. Dance, S. D. R. Williamson, N. M. H. Butler, R. Wilson, R. Capdessus, C. Armstrong, J. S. Green, S. J. Hawkes, P. Martin, W. Q. Wei, S. R. Mirfayzi, X. H. Yuan, S. Kar, M. Borghesi, R. J. Clarke, D. Neely, and P. McKenna, Near-100 mev protons via a laser-driven transparency-enhanced hybrid acceleration scheme, Nat. Commun. 9, 724 (2018).

[13] M. Barberio, M. Scisciò, S. Vallières, F. Cardelli, S. N. Chen, G. Famulari, T. Gangolf, G. Revet, A. Schiavi, M. Senzacqua, and P. Antici, Laser-accelerated particle beams for stress testing of materials, Nat. Commun. 9, 372 (2018).

[14] A. Y. Faenov et al., Submicron ionography of nanostructures using a femtosecond-laser-driven-cluster-based source, Appl. Phys. Lett. 95, 101107 (2009).
[15] S. D. Kraft et al., Dose-dependent biological damage of tumour cells by laser-accelerated proton beams, New J. Phys. 12, 085003 (2010).

[16] M. Roth, T. E. Cowan, M. H. Key, S. P. Hatchett, C. Brown, W. Fountain, J. Johnson, D. M. Pennington, R. A. Snavely, S. C. Wilks, K. Yasuike, H. Ruhl, P. Pegoraro, S. V. Bulanov, E. M. Campbell, M. D. Perry, and H. Powell, Fast Ignition by Intense Laser-Accelerated Proton Beams, Phys. Rev. Lett. 86, 436 (2001).

[17] A. B. Zylstra, J. A. Frenje, P. E. Grabowski, C. K. Li, G. W. Collins, P. Fitzsimmons, S. Glenzer, F. Graziani, S. B. Hansen, S. X. Hu, M. G. Johnson, P. Keiter, H. Reynolds, J. R. Rygg, F. H. Séguin, and R. D. Petrasso, Measurement of Charged-Particle Stopping in Warm Dense Plasma, Phys. Rev. Lett. 114, 215002 (2015).

[18] L. Romagnani, J. Fuchs, M. Borghesi, P. Antici, P. Audebert, F. Ceccherini, T. Cowan, T. Grismayer, S. Kar, A. Macchi, P. Mora, G. Pretzler, A. Schiavi, T. Toncian, and O. Willi, Dynamics of Electric Fields Driving the Laser Acceleration of Multi-Mev Protons, Phys. Rev. Lett. 95, 195001 (2005).

[19] M. Borghesi, A. J. Mackinnon, D. H. Campbell, D. G. Hicks, S. Kar, P. K. Patel, D. Price, L. Romagnani, A. Schiavi, and O. Willi, Multi-Mev Proton Source Investigations in Ultraintense Laser-Foil Interactions, Phys. Rev. Lett. 92, 055003 (2004).

[20] P. Antici, M. Migliorati, A. Mostacci, L. Picardi, L. Palumbo, and C. Ronsivalle, A compact post-acceleration scheme for laser-generated protons, Phys. Plasmas 18, 073103 (2011).

[21] I. Hofmann, Performance of solenoids versus quadrupoles in focusing and energy selection of laser accelerated protons, Phys. Rev. Accel. Beams 16, 041302 (2013).

[22] T. Toncian, M. Borghesi, J. Fuchs, E. Dhumieres, P. Antici, P. Audebert, E. Brambrink, C. A. Cecchetti, A. Pipahl, L. Romagnani et al., Ultrafast laser-driven microlens to focus and energy-select mega-electron volt protons, Science 312, 410 (2006).

[23] S. Busold, D. Schumacher, C. Brabetz, D. Jahn, F. Kroll, O. Deppert, U. Schramm, T. E. Cowan, A. Blažević, V. Bagnoud, and M. Roth, Towards highest peak intensities for ultra-short mev-range ion bunches, Sci. Rep. 5, 12459 (2015).

[24] D. Jahn, D. Schumacher, C. Brabetz, J. Ding, S. Weih, F. Kroll, F. Brack, U. Schramm, A. Blažević, and M. Roth, First application studies at the laser-driven light beamline: Improving proton beam homogeneity and imaging of a solid target, Nucl. Instrum. Methods Phys. Res., Sect. A 909, 173 (2018).

[25] F. Romano et al., The elimed transport and dosimetry beamline for laser-driven ion beams, Nucl. Instrum. Methods Phys. Res., Sect. A 829, 153 (2016).

[26] U. Masood, T. E. Cowan, W. Enghardt, K. M. Hofmann, L. Karsch, F. Kroll, U. Schramm, J. J. Wilkens, and J. Pawelke, A light-weight compact proton gantry design with a novel dose delivery system for broad-energetic laser-accelerated beams, Physics in Medicine \& Biology 62, 5531 (2017). 
[27] J. G. Zhu, M. J. Wu, Q. Liao, Y. X. Geng, K. Zhu, C. C. Li, X. H. Xu, D. Y. Li, Y. R. Shou, T. Yang, P. J. Wang, D. H. Wang, J. J. Wang, C. E. Chen, X. T. He, Y. Y. Zhao, W. J. Ma, H. Y. Lu, T. Tajima, C. Lin, and X. Q. Yan, Experimental demonstration of a laser proton accelerator with accurate beam control through image-relaying transport, Phys. Rev. Accel. Beams 22, 061302 (2019).

[28] J. van Tilborg, S. Steinke, C. G. R. Geddes, N. H. Matlis, B. H. Shaw, A. J. Gonsalves, J. V. Huijts, K. Nakamura, J. Daniels, C. B. Schroeder, C. Benedetti, E. Esarey, S. S. Bulanov, N. A. Bobrova, P. V. Sasorov, and W. P. Leemans, Active Plasma Lensing for Relativistic Laser-PlasmaAccelerated Electron Beams, Phys. Rev. Lett. 115, 184802 (2015).

[29] M. Wu, J. Zhu, D. Li, T. Yang, Q. Liao, Y. Geng, X. Xu, C. Li, Y. Shou, Y. Zhao, Y. Lu, H. Lu, W. Ma, C. Lin, K. Zhu, and $\mathrm{X}$. Yan, Collection and focusing of laser accelerated proton beam by an electromagnetic quadrupole triplet lens, Nucl. Instrum. Methods Phys. Res., Sect. A 955, 163249 (2020).

[30] M. Reiser, Theory and Design of Charged Particle Beams (Lawrence Berkeley Laboratory, Berkeley, California, 1995), vol. 48.

[31] D. Welch, D. Rose, T. Genoni, S. Yu, and J. Barnard, Simulations of neutralized final focus, Nucl. Instrum. Methods Phys. Res., Sect. A 544, 236 (2005).

[32] T. Tajima and J. M. Dawson, Laser Electron Accelerator, Phys. Rev. Lett. 43, 267 (1979).

[33] T. Hosokai, M. Kando, H. Dewa, H. Kotaki, S. Kondo, N. Hasegawa, K. Nakajima, and K. Horioka, Optical guidance of terrawatt laser pulses by the implosion phase of a fast z-pinch discharge in a gas-filled capillary, Opt. Lett. 25, 10 (2000).

[34] R. Pompili, G. Castorina, M. Ferrario, A. Marocchino, and A. Zigler, Guiding of charged particle beams in curved capillary-discharge waveguides, AIP Adv. 8, 015326 (2018).

[35] P. Chen, K. Oide, A. M. Sessler, and S. S. Yu, Plasmabased Adiabatic Focuser, Phys. Rev. Lett. 64, 1231 (1990).

[36] W. K. H. Panofsky and W. R. Baker, A focusing device for the external 350-mev proton beam of the 184-inch cyclotron at berkeley, Rev. Sci. Instrum. 21, 445 (1950).

[37] F. Dothan, H. Riege, E. Boggasch, and K. Frank, Dynamics of a $\mathrm{z}$ pinch for focusing high-energy charged particles, J. Appl. Phys. 62, 3585 (1987).

[38] A. Tauschwitz, E. Boggasch, D. H. H. Hoffmann, M. de Magistris, U. Neuner, M. Stetter, R. Tkotz, T. Wagner, W. Seelig, and H. Wetzler, The plasma lens solution for heavyion beam focusing, Il Nuovo Cimento Soc. Ital. Fis. (19651970) 106A, 1733 (1993).

[39] E. Boggasch, B. Heimrich, and D. Hoffmann, Focusing behaviour of plasma lenses compared to conventional quadrupole systems, Nucl. Instrum. Methods Phys. Res., Sect. A 336, 438 (1993).

[40] P. Vrba and M. Vrbová, Z-pinch evolution in capillary discharge, Contrib. Plasma Phys. 40, 581 (2000).

[41] D. J. Spence and S. M. Hooker, Investigation of a hydrogen plasma waveguide, Phys. Rev. E 63, 015401(R) (2000).

[42] A. J. Gonsalves et al., Petawatt Laser Guiding and Electron Beam Acceleration to $8 \mathrm{Gev}$ in a Laser-Heated Capillary
Discharge Waveguide, Phys. Rev. Lett. 122, 084801 (2019).

[43] Y. Ehrlich, C. Cohen, A. Zigler, J. Krall, P. Sprangle, and E. Esarey, Guiding of High Intensity Laser Pulses in Straight and Curved Plasma Channel Experiments, Phys. Rev. Lett. 77, 4186 (1996).

[44] J. P. Palastro, D. Kaganovich, B. Hafizi, Y.-H. Chen, L. A. Johnson, J. R. Peñano, M. H. Helle, and A. A. Mamonau, Synchrotron radiation from a curved plasma channel laser wakefield accelerator, Phys. Plasmas 24, 033119 (2017).

[45] J. Luo, M. Chen, W. Y. Wu, S. M. Weng, Z. M. Sheng, C. B. Schroeder, D. A. Jaroszynski, E. Esarey, W. P. Leemans, W. B. Mori, and J. Zhang, Multistage Coupling of Laser-Wakefield Accelerators with Curved Plasma Channels, Phys. Rev. Lett. 120, 154801 (2018).

[46] M. Chen, J. Luo, F.-Y. Li, F. Liu, Z.-M. Sheng, and J. Zhang, Tunable synchrotron-like radiation from centimeter scale plasma channels, Light Sci. Appl. 5, e16015 (2016).

[47] R. Pompili et al., Experimental characterization of active plasma lensing for electron beams, Appl. Phys. Lett. 110, 104101 (2017).

[48] C. A. Lindstrøm, E. Adli, G. Boyle, R. Corsini, A. E. Dyson, W. Farabolini, S. M. Hooker, M. Meisel, J. Osterhoff, J.-H. Röckemann, L. Schaper, and K. N. Sjobak, Emittance Preservation in an Aberration-free Active Plasma Lens, Phys. Rev. Lett. 121, 194801 (2018).

[49] S. Y. Lee, Accelerator Physics, 2nd ed. (World Scientific, Singapore, 2004).

[50] R. B. White, Theory of Tokamak Plasmas (Elsevier Science Ltd, Oxon, 1990).

[51] L. N. Brouwer, Canted-cosine-theta superconducting accelerator magnets for high energy physics and ion beam cancer therapy, Ph.D. thesis, University of California, Berkeley, 2015.

[52] T. Peter and J. Meyer-ter-Vehn, Energy loss of heavy ions in dense plasma. i. linear and nonlinear vlasov theory for the stopping power, Phys. Rev. A 43, 1998 (1991).

[53] J. D’Avanzo, I. Hofmann, and M. Lontano, Charge dependence of nonlinear stopping power, Nucl. Instrum. Methods Phys. Res., Sect. A 415, 632 (1998).

[54] H. B. Nersisyan, Stopping of charged particles in a magnetized classical plasma, Phys. Rev. E 58, 3686 (1998).

[55] R. Kowalewicz, E. Boggasch, D. Hoffmann, J. Jacoby, W. Laux, C. Stöckl, K. Weyrich, and S. Miyamoto, Enhanced energy loss of heavy ions passing a fully ionized hydrogen plasma, Laser Part. Beams 14, 599 (1996).

[56] S. Chandrasekhar, Stochastic problems in physics and astronomy, Rev. Mod. Phys. 15, 1 (1943).

[57] F. Bloch, Zur bremsung rasch bewegter teilchen beim durchgang durch materie, Ann. Phys. (Berlin) 408, 285 (1933).

[58] S. Robertson, Collective Focusing of an Intense Ion Beam, Phys. Rev. Lett. 48, 149 (1982).

[59] J. van Tilborg, S. K. Barber, H.-E. Tsai, K. K. Swanson, S. Steinke, C. G. R. Geddes, A. J. Gonsalves, C. B. Schroeder, E. Esarey, S.S. Bulanov, N. A. Bobrova, P. V. Sasorov, and W. P. Leemans, Nonuniform discharge currents in active plasma lenses, Phys. Rev. Accel. Beams 20, 032803 (2017). 
[60] N. A. Bobrova, A. A. Esaulov, J. I. Sakai, P. V. Sasorov, D. J. Spence, A. Butler, S. M. Hooker, and S. V. Bulanov, Simulations of a hydrogen-filled capillary discharge waveguide, Phys. Rev. E Stat. Nonlin. Soft Matter Phys. 65, 016407 (2001).

[61] M. de Magistris, L. De Menna, G. Miano, and C. Serpico, An analytical approach to optical properties of plasma lenses with a non-linear magnetic field profile, Fusion Eng. Des. 32-33, 377 (1996).

[62] J. van Tilborg, S. K. Barber, C. Benedetti, C. B. Schroeder, F. Isono, H.-E. Tsai, C. G. R. Geddes, and W. P. Leemans, Comparative study of active plasma lenses in high-quality electron accelerator transport lines, Phys. Plasmas 25, 056702 (2018).

[63] R. Pompili, E. Chiadroni, A. Cianchi, A. Del Dotto, L. Faillace, M. Ferrario, P. Iovine, and M. R. Masullo, Plasma lens-based beam extraction and removal system for plasma wakefield acceleration experiments, Phys. Rev. Accel. Beams 22, 121302 (2019).

[64] L.-Y. Zhang, X.-Y. Zhao, X. Qi, W.-S. Duan, and L. Yang, Particle-in-cell simulations of the long proton beam focusing in background plasmas, Laser Part. Beams 34, 299-305 (2016).

[65] I. D. Kaganovich, G. Shvets, E. Startsev, and R. C. Davidson, Nonlinear charge and current neutralization of an ion beam pulse in a pre-formed plasma, Phys. Plasmas 8, 4180 (2001).

[66] B. Blue, Plasma wakefield acceleration of an intense positron beam, Ph.D. thesis, Stanford University, Stanford, 2004.

[67] R. Pompili et al., Focusing of High-Brightness Electron Beams with Active-Plasma Lenses, Phys. Rev. Lett. 121, 174801 (2018).
[68] T. D. Arber, K. Bennett, C.S. Brady, A. LawrenceDouglas, M. G. Ramsay, N. J. Sircombe, P. Gillies, R. G. Evans, H. Schmitz, A. R. Bell, and C. P. Ridgers, Contemporary particle-in-cell approach to laser-plasma modelling, Plasma Phys. Controlled Fusion 57, 113001 (2015).

[69] E. M. Bringa and N. R. Arista, Collective effects in the energy loss of ion beams in fusion plasmas, Phys. Rev. E 52, 3010 (1995).

[70] M. J. Wu, D. Y. Li, J. G. Zhu, T. Yang, X. Y. Hu, Y. X. Geng, K. Zhu, M. J. Easton, Y. Y. Zhao, A. L. Zhang, H. Y. Lu, W. J. Ma, C. Lin, and X. Q. Yan, Emittance measurement along transport beam line for laser driven protons, Phys. Rev. Accel. Beams 23, 031302 (2020).

[71] D. G. Luenberger, Y. Ye et al., Linear and Nonlinear Programming (Springer, New York, 1984), Vol. 2.

[72] F. L. Zheng, H. Y. Wang, X. Q. Yan, T. Tajima, M. Y. Yu, and X. T. He, Sub-tev proton beam generation by ultraintense laser irradiation of foil-and-gas target, Phys. Plasmas 19, 023111 (2012).

[73] M. A. Bake, A. Aimidula, F. Xiaerding, and R. Rashidin, Enhanced proton acceleration by intense laser interaction with an inverse cone target, Phys. Plasmas 23, 083107 (2016).

[74] H.-Y. Ban, Y.-J. Gu, Q. Kong, Y.-Y. Li, Z. Zhu, and S. Kawata, Quasi-monoenergetic tens-of-MeV proton beams by a laser-illuminated funnel-like target, Chin. Phys. Lett. 29, 035202 (2012).

[75] Z. M. Zhang, X. T. He, Z. M. Sheng, and M. Y. Yu, Hundreds mev monoenergetic proton bunch from interaction of $102021 \mathrm{w} / \mathrm{cm} 2$ circularly polarized laser pulse with tailored complex target, Appl. Phys. Lett. 100, 134103 (2012). 